\title{
Frequency of Human Papilloma Virus Genotypes in Patient Samples From Isfahan (Iran) Using Polymerase Chain Reaction Reverse Dot Blot Method
}

\author{
Behnam Toghani Pour, ${ }^{1}$ Seyed Masoud Hosseini, ${ }^{1,}$ and Mohammad Taghi Kardi ${ }^{2}$ \\ ${ }^{1}$ Department of Microbiology, Faculty of Biological Sciences, Shahid Beheshti University, Tehran, Iran \\ ${ }^{2}$ Mahdieh Medical Laboratory, Isfahan, Iran \\ "Corresponding author: Seyed Masoud Hosseini, PhD, Department of Microbiology, Faculty of Biological Sciences, Shahid Beheshti University, Tehran, Iran. Tel: +98-21-22902721, \\ E-mail: Ma_Hosseini@sbu.ac.ir
}

Received 2016 December 13; Revised 2017 February 04; Accepted 2017 February 25.

\begin{abstract}
Background: Human papilloma virus (HPV) is the most common sexually transmitted viral infection worldwide. On the basis of HPV clinical associations, this disease is divided to 2 types; high risk and low risk. high risk HPV (HR HPV) is the causative agent of cervical intraepithelial Neoplasia (CIN) and cervical cancer. low risk HPV (LR HPV) is the causative agent of diseases, such as anogenital warts and oral papillomas. Polymerase chain reaction (PCR) is one of the molecular diagnostic methods for detection of HPV DNA that has many advantages.

Objectives: This study aimed at exploring the frequency of HPV genotypes using polymerase chain reaction reverse dot blot (PCR RDB) method on cervix and wart samples obtained from patients in Isfahan, Iran.

Methods: The population included 111 females and 7 males. These samples were collected during 2011 and 2013. DNA was extracted from these samples and PCR was performed using reverse dot blot (RDB) method to detect HPV types.

Results: With this molecular diagnostic method, different HPV types were detected in 45 samples out of 118 . In 18 samples, coinfection with different HPV types was detected. Data analysis exhibited a significant correlation between age and HR HPV infection (P value < 0.05). However, no significant relationship was detected between LR HPV and age (P value > 0.05). In this study, among all HPV types, HPV 16 was detected more frequently than the other HPV types.

Conclusions: The PCR-RDB method has the ability to diagnose HPV infection by screening Pap smear samples and to determine the risk factor of detected HPV genotypes. This study showed that HPV16 was 1 of the most common agents in Iran, similar to other countries.
\end{abstract}

Keywords: Papillomavirus Infections, Polymerase Chain Reaction Reverse Dot Blot Method, Warts

\section{Background}

Human papilloma virus (HPV) infection is one of the most common sexually transmitted diseases worldwide. Up to $70 \%$ of sexually active females may become infected with HPV during their life-time, around the world (1). Human papilloma virus has more than 100 genotypes, nonenveloped icosahedral shell, capsid of 72 pentamers, and double stranded DNA genome (1). Corresponding to their association with disease types, papillomaviruses are classified to High Risk (HR) and Low Risk (LR) types. High RiskHPV types (HPV 16, 18, 31, 35, 39, 45, 51, 52, 56, 39, 45, 51, 52, $56,59,66,68,69$, and 73 ) are often associated with high grade lesions and invasive cancer, whereas, the LR HPV types (i.e. HPV 6, 11, 40, 42, 43, 44, 54, 61, 70, 72, and 81) are mainly found in low grade lesions, genital or skin warts, and condylomaaccuminata (2). Persistent infection of specific types of HR-HPVs, particularly types 16 and 18, which possess the E6 and E7 oncogenes, will consequently lead to development of Cervical Intraepithelial Neoplasia (CIN) and cervical cancer (2-4).

The PCR technique, which is based on detection of HPV's DNA, is an alternative technique to HPV testing. The advantages of PCR are rapidity, sensitivity, ability to detect nonviable virus, and potential elimination of contaminating microorganisms. Nowadays, HPV diagnostic methods are developed based on diagnosing virus nucleic acid (5).

\section{Objectives}

In the current study, reverse dot blot PCR (RDB-PCR) was used to detect HPV genotypes in positive HPV samples, which could help with diagnosis and consequently prevent cancers such as genital cancer by detecting high risk genotypes. 


\section{Methods}

The current study was carried out on patients, who were referred to 2 hospitals and a specialized laboratory in Isfahan during years 2011 and 2013. This research was done on 111 cervix samples with liquid-based cytology method. Also, 7 wart samples were collected from male cases.

\subsection{DNA Genome Extraction and DNA Amplification}

For DNA extraction, RIBO-Prep nucleic acid extraction kit variant 100/Russia was used and the PCR product was generated with a primer labeled by biotin.

\subsection{Reverse Dot Blot Method}

The HPV types were recognized with the INNOGENETICS kit (INNO-LIPA HPV Genotyping Extra). Enzymatic reaction, which generates color, occurred when the PCR product was linked with a probe on a solid surface. Streptavidin along with alkaline phosphatase were used to identify the PCR products by producing color.

In this procedure, $10 \mu \mathrm{L}$ of PCR product labeled with biotin was added to a denaturing solution at $35^{\circ} \mathrm{C}$ for $10 \mathrm{~min}$ utes. In the next step, nitrocellulose strip was added and placed at $49^{\circ} \mathrm{C}$ for 1 hour until hybridization was accomplished. After this stage, washing was done twice with the staining solution followed by washing twice with $2 \mathrm{~mL}$ of rinse solution and then placed at $30^{\circ} \mathrm{C}$ for 30 minutes with conjugating solution. A further wash was done twice with $2 \mathrm{~mm}$ of rinse solution. Finally, the substrate was kept at $35^{\circ} \mathrm{C}$ for 30 minutes to react with alkaline phosphates until staining had occurred. After staining, nitrocellulose strip was compared with the diagnosis strip.

\section{Results}

For recognizing HPV types, the assigned numbers on cellulose strip were compared with the HPV genotyping list (Figure 1). Among the 111 cervix samples and 7 wart samples, 41 and 4 cases had positive HPV test results, respectively. The detected genotypes were HR-HPV types, 16, 18, 31, $32,33,39,45,51,52,56,58,59,66$, and 68, and LR-HPV 1, 6, 11,26 , and 44, which are stated in Table 1 . Some cases had co-infection with different HPV types. Statistical analysis was performed with the SPSS software, version 20. Pearson correlation coefficient exhibited a significant association between the HPV test (-0.361) and age (95\% confidence interval, $\mathrm{P}=0.001)$, also it exhibited a significant association between HR HPV type (-0.273) and age (95\% CI, P = 0.009). However, no significant correlation was found between LR HPV types (-153) and age (95\% CI, P = 0.147).

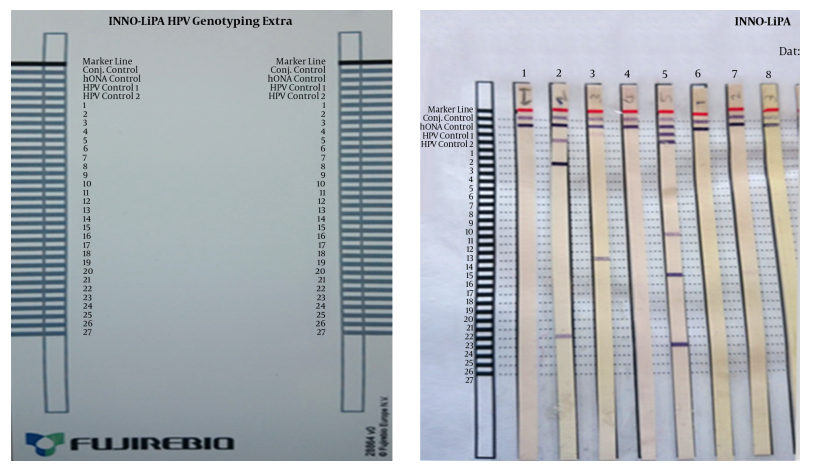

Figure 1. Nitrocellulose Strips After Staining

\section{Discussion}

The annual incidence of cervical cancer worldwide is more than 450,000 cases. Although the mortality rate has declined over the past 30 years, almost 200,000 deaths from cervical cancer occur each year. In the US, more than 12,000 new cases of cervical cancer are diagnosed and 400 deaths are reported, annually. Human Papilloma Virus is considered as the main cause of malignant lesions and cervical cancer. Furthermore, HPV 16 and HPV 18 infection are responsible for about $70 \%$ of all cervical cancers, worldwide (6-8).

In European countries with the use of sanitation, pregnancy prevention and considering the risks of unsafe sex, the spreading rate of the disease is declining. On the other hand, in Iran due to lack of hygiene, the disease is increasing, especially high risk types. It is even detected in males as well. It is noteworthy that HPV infection in patients with cervical cancer is $76 \%$, while in healthy Iranian females, it has been reported at about $7 \%$. Cervical cancer rates increase in the community above age 30 , and the prevalence is maximum between 55 and 65 years of age. As Iran is a developing country and also since it is exposed to cancer threats, regular screening is required for preventing cancer in the Iranian community.

The best way for preventing of cervical cancer in females is a screening program with cytology test and molecular diagnostic methods, with high sensitivity instead of conventional methods. Finally, prevention is required with effective vaccines, according to dominant genotypes in the area and treatment. Currently, 2 prophylactic vaccines, Cervarix and Gardasil, are licensed for use in many countries, worldwide. Cervarix is a bivalent vaccine containing Virus-Like particles (VLPs) of L1 from HPV16 and 18 that are the two virus types that cause most cervical cancers and other HPV-associated cancers, worldwide. Gardasil is a quadrivalent vaccine containing VLPs of HPV 6 
Table 1. Human Papilloma Virus Types Distribution in Female Cervix and Male Wart Samples

\begin{tabular}{|c|c|c|c|c|c|c|c|c|c|}
\hline No. & Age & Gender & HP HPV Type & LR HPV Type & No. & Age & Gender & HP HPV Type & LR HPV Type \\
\hline 1 & 20 & \multirow{23}{*}{ female } & - & 1 & 24 & 45 & \multirow{18}{*}{ female } & 16,66 & - \\
\hline 2 & 25 & & - & 6 & 25 & 41 & & 16 & - \\
\hline 3 & 25 & & & 6 & 26 & 26 & & $45,18,51$ & - \\
\hline 4 & 33 & & 33,31 & - & 27 & 33 & & 31,33 & - \\
\hline 5 & 27 & & 16 & - & 28 & 32 & & 51 & - \\
\hline 6 & 31 & & 45 & - & 29 & 26 & & $31,33,52,58$ & - \\
\hline 7 & 25 & & 16 & - & 30 & - & & 16 & - \\
\hline 8 & - & & 56,58 & - & 31 & 24 & & $33,31,59,52$ & 11 \\
\hline 9 & 33 & & 16 & - & 32 & 28 & & 68,51 & - \\
\hline 10 & 29 & & 16 & - & 33 & - & & $18,39,68,45$ & 51 \\
\hline 11 & 26 & & 16 & - & 34 & - & & - & 6 \\
\hline 12 & - & & 16 & - & 35 & - & & $39,68,33,18$ & - \\
\hline 13 & - & & 16 & - & 36 & 44 & & 16 & - \\
\hline 14 & - & & 16 & - & 37 & 35 & & 31,33 & - \\
\hline 15 & - & & $31,32,58,52$ & - & 38 & 24 & & 45 & - \\
\hline 16 & 34 & & $31,33,58$ & - & 39 & 38 & & - & 6 \\
\hline 17 & 26 & & $51,52,58$ & - & 40 & - & & - & 6 \\
\hline 18 & - & & 16 & - & 41 & 29 & & - & 26 \\
\hline 19 & - & & 18 & - & 42 & 22 & \multirow{5}{*}{ male } & 6 & 51 \\
\hline 20 & 26 & & $31,33,51,52$ & - & 43 & 32 & & 6 & - \\
\hline 21 & 38 & & 18 & - & 44 & 36 & & 18 & - \\
\hline 22 & - & & $18,56,58$ & - & 45 & 35 & & 6 & 51 \\
\hline 23 & 27 & & 16 & - & & & & & \\
\hline
\end{tabular}

and 11, which together cause most genital warts, in addition to HPV 16 and HPV $18(9,10)$.

\section{Footnotes}

Authors' Contribution: The authors had equal contribution of this experimental study.

Financial Disclosure: There was no conflict of interest for conducting this study.

Funding/Support: This study was funded by the research and technical deputy of Shahid Bebeshti University.

\section{References}

1. Sohrabi A, Mirab-Samiee S, Modarresi MH, Izadimood N, Azadmanesh $\mathrm{K}$, Rahnamaye-Farzami M. Development of in-house multiplex real time PCR for human papillomavirus genotyping in Iranian women with cervical cancer and cervical intraepithelial neoplasia. Asian Pac J Cancer Prev. 2014;15(15):6257-61. [PubMed: 25124608].

2. Bharti AC, Shukla S, Mahata S, Hedau S, Das BC. Anti-human papillomavirus therapeutics: facts \& future. Indian J Med Res. 2009;130(3):296-310. [PubMed: 19901439].

3. Jia H, Wang X, Long Z, Li L. Human papillomavirus infection and cervical dysplasia in female sex workers in Northeast China: an observational study. BMC Public Health. 2015;15:695. doi: 10.1186/s12889-0152066-x. [PubMed: 26202513].
4. Raji N, Sadeghizadeh M, Tafreshi KN, Jahanzad E. Detection of human Papillomavirus 18 in cervical cancer samples using PCR-ELISA(DIAPOPS). Iran J Microbiol. 2011;3(4):177-82. [PubMed: 22530085].

5. Abreu AL, Souza RP, Gimenes F, Consolaro ME. A review of methods for detect human Papillomavirus infection. Virol J. 2012;9:262. doi: 10.1186/1743-422X-9-262. [PubMed: 23131123].

6. Jemal A, Bray F, Center MM, Ferlay J, Ward E, Forman D. Global cancer statistics. CA Cancer J Clin. 2011;61(2):69-90. doi: 10.3322/caac.20107. [PubMed: 21296855].

7. Laudadio J. Human papillomavirus detection: testing methodologies and their clinical utility in cervical cancer screening. Adv Anat Pathol. 2013;20(3):158-67. doi: 10.1097/PAP.0b013e31828d1893. [PubMed: 23574772].

8. Wheeler CM, Hunt WC, Joste NE, Key CR, Quint WG, Castle PE. Human papillomavirus genotype distributions: implications for vaccination and cancer screening in the United States. J Natl Cancer Inst. 2009;101(7):475-87. doi:10.1093/jnci/djn510. [PubMed: 19318628].

9. Leung TF, Liu AP, Lim FS, Thollot F, Oh HM, Lee BW, et al. Comparative immunogenicity and safety of human papillomavirus (HPV)16/18 AS04-adjuvanted vaccine and HPV-6/11/16/18 vaccine administered according to 2- and 3-dose schedules in girls aged 9-14 years: Results to month 12 from a randomized trial. Hum Vaccin Immunother. 2015;11(7):1689-702. doi: 10.1080/21645515.2015.1050570. [PubMed: 26062002].

10. Schiller JT, Muller M. Next generation prophylactic human papillomavirus vaccines. Lancet Oncol. 2015;16(5):e217-25. doi: 10.1016/S14702045(14)71179-9. [PubMed: 25943066]. 